\title{
AN OPTIMAL APPROXIMATION FORMULA FOR FUNCTIONS WITH SINGULARITIES
}

\author{
KEN'ICHIRO TANAKA, TOMOAKI OKAYAMA, AND MASAAKI SUGIHARA
}

\begin{abstract}
We propose an optimal approximation formula for analytic functions that are defined on a complex region containing the real interval $(-1,1)$ and possibly have algebraic singularities at the endpoints of the interval. As a space of such functions, we consider a Hardy space with the weight given by $w_{\mu}(z)=\left(1-z^{2}\right)^{\mu / 2}$ for $\mu>0$, and formulate the optimality of an approximation formula for the functions in the space. Then, we propose an optimal approximation formula for the space for any $\mu>0$ as opposed to existing results with the restriction $0<\mu<\mu_{*}$ for a certain constant $\mu_{*}$. We also provide the results of numerical experiments to show the performance of the proposed formula.
\end{abstract}

\section{INTRODUCTION}

This paper is concerned with approximation of functions by a finite number of the sampled values of them. We consider analytic functions that are defined on a complex region containing a real interval and possibly have endpoint singularities on the interval. In order to deal with such functions collectively, we consider a function space consisting of them and formulate the optimality of an approximation formula for the functions in the space. Then, we propose an optimal approximation formula for the function space.

We consider the region given by

$$
\Lambda_{d}=\left\{z \in \mathbf{C}|| \arg \left(\frac{1+z}{1-z}\right) \mid<d\right\},
$$

which satisfies $\Lambda_{d} \cap \mathbf{R}=(-1,1)$. In order to deal with analytic functions on $\Lambda_{d}$ with algebraic singularities at the endpoints \pm 1 , we consider the function space given by

$$
\boldsymbol{H}^{\infty}\left(\Lambda_{d}, w_{\mu}\right)=\left\{f: \Lambda_{d} \rightarrow \mathbf{C} \mid f \text { is analytic in } \Lambda_{d} \text { and } \sup _{z \in \Lambda_{d}}\left|\frac{f(z)}{w_{\mu}(z)}\right|<\infty\right\}
$$

where $\mu$ is a positive number and $w_{\mu}(z)=\left(1-z^{2}\right)^{\mu / 2}$. This space has been studied as a fundamental space for the sinc numerical methods [6, 8, which are the numerical methods based on the approximation of functions by the sinc function (see (2.6) ). The error analysis of the sinc approximation has been performed in these decades [6, 7, 8, 10]. It is well-known that the sinc approximation has very good accuracy in $\boldsymbol{H}^{\infty}\left(\Lambda_{d}, w_{\mu}\right)$.

Date: October 22, 2016.

2010 Mathematics Subject Classification. Primary 65D15; Secondary 41A25.

Key words and phrases. Hardy space, endpoint singularity, optimal approximation, Ganelius sampling points. 
Besides the studies of such concrete formulas in $\boldsymbol{H}^{\infty}\left(\Lambda_{d}, w_{\mu}\right)$, there are several analyses of the errors of optimal formulas in spaces of analytic functions like $\boldsymbol{H}^{\infty}\left(\Lambda_{d}, w_{\mu}\right)$. In the literatures [1, 5, 9, 12, the authors have estimated the optimal errors in Hardy spaces with preassigned decay rates. In particular, Sugihara 9 has given a lower bound of the optimal error in $\boldsymbol{H}^{\infty}\left(\Lambda_{d}, w_{\mu}\right)$ and revealed that the sinc approximation is near optimal in the space. In order to formulate the optimality of an approximation formula for the functions in $\boldsymbol{H}^{\infty}\left(\Lambda_{d}, w_{\mu}\right)$, he considered all the possible $n$-point approximation formulas in the space and the norms of their error operators. Then, he defined the minimum error norm $E_{n}^{\min }\left(\boldsymbol{H}^{\infty}\left(\Lambda_{d}, w_{\mu}\right)\right)$ by the minimum of the norms. Furthermore, he also considered the error norm of the sinc approximation on $\boldsymbol{H}^{\infty}\left(\Lambda_{d}, w_{\mu}\right)$, denoted by $E_{n}^{\operatorname{sinc}}\left(\boldsymbol{H}^{\infty}\left(\Lambda_{d}, w_{\mu}\right)\right)$, and has shown that

$c^{\prime \prime} \exp \left(-c_{2} \sqrt{n}\right) \leq E_{n}^{\min }\left(\boldsymbol{H}^{\infty}\left(\Lambda_{d}, w_{\mu}\right)\right) \leq E_{n}^{\operatorname{sinc}}\left(\boldsymbol{H}^{\infty}\left(\Lambda_{d}, w_{\mu}\right)\right) \leq c^{\prime} \sqrt{n} \exp \left(-c_{1} \sqrt{n}\right)$, where $c^{\prime}, c^{\prime \prime}, c_{1}$, and $c_{2}$ are positive constants with $c_{1}<c_{2}$ (see (2.9)).

However, finding an explicit approximation formula attaining $E_{n}^{\min }\left(\boldsymbol{H}^{\infty}\left(\Lambda_{d}, w_{\mu}\right)\right)$ has been an open problem so far whereas the exact order of $E_{n}^{\min }\left(\boldsymbol{H}^{\infty}\left(\Lambda_{d}, w_{\mu}\right)\right)$ with respect to $n$ is known in some restricted case. Recently, in the restricted case that $0<\mu<\min \{2, \pi / d\}$, Ushima et al. [11] have proposed an optimal formula by using the technique of Jang and Haber [3], in which they employ a modification of the sampling points given by Ganelius [2]. The restriction $0<\mu<\min \{2, \pi / d\}$ is owing to the assumption $r<1$ in the Ganelius theorem [3, Lemma 1], which plays an important role for the error estimate of the formula. In this paper, we remove this restriction and propose an optimal formula for any $\mu>0$ by generalizing the formula in 11 .

The rest of this paper is organized as follows. In Section 2, we list mathematical tools for setting the framework for approximation of the functions in $\boldsymbol{H}^{\infty}\left(\Lambda_{d}, w_{\mu}\right)$. We give the more precise explanations of the region $\Lambda_{d}$, space $\boldsymbol{H}^{\infty}\left(\Lambda_{d}, w_{\mu}\right)$, and the notion of the optimal approximation in $\boldsymbol{H}^{\infty}\left(\Lambda_{d}, w_{\mu}\right)$. Furthermore, we review some existing results about the estimate of $E_{n}^{\min }\left(\boldsymbol{H}^{\infty}\left(\Lambda_{d}, w_{\mu}\right)\right)$. In Section 3, we present our new formula and show its error estimate in Theorem 3.3. By combining this theorem and the existing result giving the lower estimate of $E_{n}^{\min }\left(\boldsymbol{H}^{\infty}\left(\Lambda_{d}, w_{\mu}\right)\right)$, we show the optimality of the proposed formula. The proof of Theorem 3.3 is owing to three lemmas, whose proofs are presented in Section 4. The last one of them, Lemma 3.6, is proven by being reduced to Theorem 4.2, a generalization of the Ganelius theorem without the assumption $r<1$. This theorem is proven in Appendix B, In Section 5 we present some numerical results showing the performance of our formula. Finally, we conclude this paper in Section 6 .

\section{Mathematical preliminaries AND EXISting Results}

2.1. Function space $\boldsymbol{H}^{\infty}\left(\Lambda_{d}, w_{\mu}\right)$. For a real number $d$ with $0<d<\pi$, we consider the strip region $\mathcal{D}_{d}:=\{\zeta \in \mathbf{C}|| \operatorname{Im} \zeta \mid<d\}$. Then, we define a region $\Lambda_{d}$ by

$$
\Lambda_{d}:=\left\{z \in \mathbf{C} \mid z=\tanh (\zeta / 2), \zeta \in \mathcal{D}_{d}\right\},
$$

and set the counterclockwise direction to its boundary $\partial \Lambda_{d}$. The region $\Lambda_{d}$ can be written in the form

$$
\Lambda_{d}=\left\{z \in \mathbf{C}|| \arg \left(\frac{1+z}{1-z}\right) \mid<d\right\}
$$


Furthermore, the region $\Lambda_{d}$ is symmetric with respect to the real axis and satisfies $\Lambda_{d} \cap \mathbf{R}=(-1,1)$. The upper half part of $\Lambda_{d}$ coincides with the intersection of the upper half plane and the open disc with center $(-\mathrm{i} / \tan d)$ and radius $1 / \sin d$. The intersection of the boundary $\partial \Lambda_{d}$ and the imaginary axis consists of $( \pm \mathrm{i} \tan (d / 2))$. In particular, the region $\Lambda_{d}$ becomes an eye-shaped region if $0<d<\pi / 2$, a unit disc if $d=\pi / 2$, and the entire complex plane if $d \rightarrow \pi$. As an example, we show the region $\Lambda_{\pi / 4}$ in Figure 1

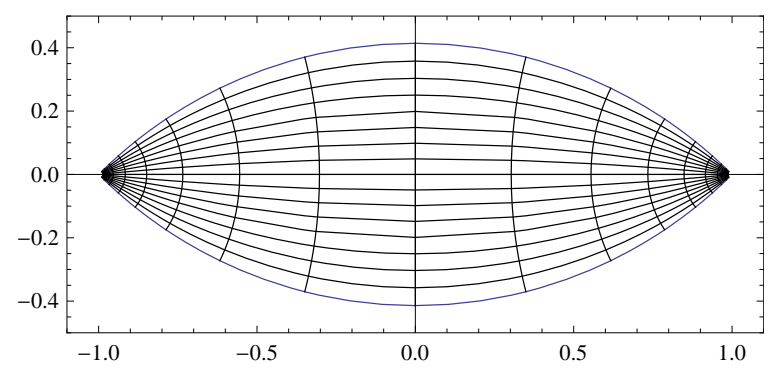

Figure 1. Region $\Lambda_{\pi / 4}$.

Throughout this paper, we consider approximation of analytic functions defined on $\Lambda_{d}$ that possibly have algebraic singularities at the endpoints \pm 1 . Accordingly, we introduce a function space of such functions as below.

Definition 2.1. Let $\mu$ be a positive number and let

$$
w_{\mu}(z):=\left(1-z^{2}\right)^{\mu / 2} .
$$

We define a function space $\boldsymbol{H}^{\infty}\left(\Lambda_{d}, w_{\mu}\right)$ by

$$
\boldsymbol{H}^{\infty}\left(\Lambda_{d}, w_{\mu}\right):=\left\{f: \Lambda_{d} \rightarrow \mathbf{C} \mid f \text { is analytic in } \Lambda_{d} \text { and }\|f\|<\infty\right\},
$$

where

$$
\|f\|:=\sup _{z \in \Lambda_{d}}\left|\frac{f(z)}{w_{\mu}(z)}\right| .
$$

Remark 2.2. A function $f \in \boldsymbol{H}^{\infty}\left(\Lambda_{d}, w_{\mu}\right)$ satisfies

$$
|f(z)| \leq\|f\|\left|\left(1-z^{2}\right)^{\mu / 2}\right|
$$

for any $z \in \Lambda_{d}$. Therefore, the function $f$ tends to zero with order $\mathrm{O}\left((1 \pm z)^{\mu / 2}\right)$ as $z \rightarrow \mp 1$.

2.2. Minimum error norm $E_{n}^{\min }\left(\boldsymbol{H}^{\infty}\left(\Lambda_{d}, w_{\mu}\right)\right)$. For $f \in \boldsymbol{H}^{\infty}\left(\Lambda_{d}, w_{\mu}\right)$, we consider all the possible $n$-point approximation formulas written in the form

$$
f(x) \approx \sum_{j=1}^{\ell} \sum_{k=0}^{m_{j}-1} f^{(k)}\left(a_{j}\right) \phi_{j k}(x),
$$

where $\ell$ is an integer with $1 \leq \ell \leq n,\left\{m_{j}\right\}$ is a sequence of nonnegative integers with $m_{1}+\cdots+m_{\ell}=n,\left\{a_{j}\right\}$ is a sequence of sampling points in $\Lambda_{d}$, and $\left\{\phi_{j k}\right\}$ is a 
sequence of analytic functions on $\Lambda_{d}$. Then, let $\mathcal{N}_{a_{j}, \phi_{j k}}^{\ell, m_{i}}$ denote the operator norm of the error operator associated with Formula (2.2):

$$
\mathcal{N}_{a_{j}, \phi_{j k}}^{\ell, m_{i}}:=\sup _{f \in \boldsymbol{H}^{\infty}\left(\Lambda_{d}, w_{\mu}\right)}\left[\sup _{x \in(\| \leq 1}\left|f(x)-\sum_{j=1}^{\ell} \sum_{k=0}^{m_{j}-1} f^{(k)}\left(a_{j}\right) \phi_{j k}(x)\right|\right] .
$$

We call the value $\mathcal{N}_{a_{j}, \phi_{j k}}^{\ell, m_{i}}$ the error norm of Formula (2.2) and adopt it as a criterion for evaluating the accuracy of Formula (2.2). Therefore, an approximation formula with the form in (2.2) is optimal if it achieves the infimum of the error norm $\mathcal{N}_{a_{j}, \phi_{j k}}^{\ell, m_{i}}$ over any $n$-point approximation formulas. By letting the infimum denoted by

$$
E_{n}^{\min }\left(\boldsymbol{H}^{\infty}\left(\Lambda_{d}, w_{\mu}\right)\right):=\inf _{1 \leq \ell \leq n} \inf _{\substack{m_{1}, \ldots, m_{\ell} \\ m_{1}+\cdots+m_{\ell}=n}} \inf _{a_{j} \in \Lambda_{d}} \inf _{\phi_{j k}} \mathcal{N}_{a_{j}, \phi_{j k}}^{\ell, m_{i}}
$$

we call it the minimum error norm of the $n$-point approximation in $\boldsymbol{H}^{\infty}\left(\Lambda_{d}, w_{\mu}\right)$. In the literature [9], a lower bound of $E_{n}^{\min }\left(\boldsymbol{H}^{\infty}\left(\Lambda_{d}, w_{\mu}\right)\right)$ is given.

Theorem 2.3 ([9, (a) on page 782]). The minimum error norm (2.4) is bounded from below as follows:

$$
E_{n}^{\min }\left(\boldsymbol{H}^{\infty}\left(\Lambda_{d}, w_{\mu}\right)\right) \geq c \exp (-\sqrt{\pi d \mu n / 2}),
$$

where $c$ is a positive number independent of $n$.

On the other hand, an upper bound of $E_{n}^{\min }\left(\boldsymbol{H}^{\infty}\left(\Lambda_{d}, w_{\mu}\right)\right)$ can be given by the error norm $\mathcal{N}_{a_{j}, \phi_{j k}}^{\ell, m_{i}}$ of an approximation formula that is applicable to the functions in $\boldsymbol{H}^{\infty}\left(\Lambda_{d}, w_{\mu}\right)$. It is well-known that an upper bound close to the lower bound in (2.5) is given by the sinc approximation formula with a variable transformation of a single exponential type as shown in the next subsection.

2.3. Nearly optimal formula (Sinc approximation). The $(2 N+1)$-point sinc approximation is defined by

$$
f(x) \approx \sum_{j=-N}^{N} f(\psi(j h)) S(j, h)\left(\psi^{-1}(x)\right)
$$

where

$$
\begin{aligned}
h & :=\sqrt{\frac{2 \pi d}{\mu N}}, \\
\psi(\zeta) & :=\tanh (\zeta / 2),
\end{aligned}
$$

and

$$
S(j, h)(t):=\frac{\sin [\pi(t / h-j)]}{\pi(t / h-j)} .
$$

Formula (2.6) is called the SE-Sinc formula, which has been intensively studied by Stenger et al. [4, 6, 7, 8]. Let $E_{2 N+1}^{\text {sinc }}\left(\boldsymbol{H}^{\infty}\left(\Lambda_{d}, w_{\mu}\right)\right)$ be the error norm $\mathcal{N}_{a_{j}, \phi_{j k}}^{\ell, m_{i}}$ of Formula (2.6), i.e.,

$$
E_{2 N+1}^{\operatorname{sinc}}\left(\boldsymbol{H}^{\infty}\left(\Lambda_{d}, w_{\mu}\right)\right)
$$




$$
:=\sup _{\substack{f \in \boldsymbol{H}^{\infty}\left(\Lambda_{d}, w_{\mu}\right) \\\|f\| \leq 1}}\left[\sup _{x \in(-1,1)}\left|f(x)-\sum_{j=-N}^{N} f(\psi(j h)) S(j, h)\left(\psi^{-1}(x)\right)\right|\right] .
$$

Sugihara [9] has shown the following upper bound of $E_{2 N+1}^{\text {sinc }}\left(\boldsymbol{H}^{\infty}\left(\Lambda_{d}, w_{\mu}\right)\right)$.

Theorem 2.4 (9, (a) on page 782]). The minimum error norm of the SE-Sinc formula is bounded from above as follows:

$$
E_{2 N+1}^{\text {sinc }}\left(\boldsymbol{H}^{\infty}\left(\Lambda_{d}, w_{\mu}\right)\right) \leq c^{\prime} \sqrt{N} \exp (-\sqrt{\pi d \mu N / 2}),
$$

where $c^{\prime}$ is a positive number independent of $N$.

From Theorem 2.3 with $n=2 N+1$ and Theorem 2.4 we have

$$
\begin{aligned}
c^{\prime \prime} \exp (-\sqrt{\pi d \mu N}) & \leq E_{2 N+1}^{\min }\left(\boldsymbol{H}^{\infty}\left(\Lambda_{d}, w_{\mu}\right)\right) \\
& \leq E_{2 N+1}^{\operatorname{sinc}}\left(\boldsymbol{H}^{\infty}\left(\Lambda_{d}, w_{\mu}\right)\right) \leq c^{\prime} \sqrt{N} \exp (-\sqrt{\pi d \mu N / 2})
\end{aligned}
$$

for some positive numbers $c^{\prime \prime}$ and $c^{\prime}$ independent of $N$, which gives an estimate of the order of the minimum error norm $E_{2 N+1}^{\min }\left(\boldsymbol{H}^{\infty}\left(\Lambda_{d}, w_{\mu}\right)\right)$ with respect to $N$.

Recently, the exact order of the minimum error norm is revealed by an explicit approximation formula as shown in the next subsection.

2.4. Optimal formula. Ushima et al. 11 have found out an explicit approximation formula that achieves the exact order of the minimum error norm for $\mu$ with $0<\mu<\min \{2, \pi / d\}$ by using the modified Ganelius sampling points proposed in [3, Lemma 1]. Furthermore, they have shown that

$$
E_{2 N}^{\min }\left(\boldsymbol{H}^{\infty}\left(\Lambda_{d}, w_{\mu}\right)\right) \leq C \exp (-\sqrt{\pi d \mu N})
$$

for a positive number $C$ independent of $N$, and that the RHS in (2.10) gives the exact order of $E_{2 N}^{\min }\left(\boldsymbol{H}^{\infty}\left(\Lambda_{d}, w_{\mu}\right)\right)$ by combining this inequality and Theorem 2.3 with $n=2 N$. In order to show the proposed formula in [11, we describe the definition of the modified Ganelius sampling points and a fundamental inequality relating to them, which plays an important role for the error estimate of the formula.

Definition 2.5. Let $r$ be a positive real number and let $N$ be a positive integer. Furthermore, let $N_{0}$ be defined by

$$
N_{0}:=N-\left\lceil\frac{\pi}{4} \sqrt{N r}\right\rceil,
$$

and let $\varphi$ be the function defined by

$$
\varphi(x):=\exp \left(\pi \sqrt{\frac{x}{r}}\right)
$$

for a positive number $x$. Then, the numbers $a_{k}$ defined by

$$
a_{k}:= \begin{cases}\varphi(k-1) / \varphi\left(N_{0}\right) & \left(k=1,2, \ldots, N_{0}\right) \\ \varphi(k-3 / 2) / \varphi\left(N_{0}\right) & \left(k=N_{0}+1\right) \\ 1-\frac{k-N_{0}-1}{5\left(N-N_{0}-1\right)} & \left(k=N_{0}+2, \ldots, N\right)\end{cases}
$$

are called the modified Ganelius sampling points. 
Theorem 2.6 ([3. Lemma 1]). Let $r$ be a positive real number satisfying $r<1$ and let $N$ be a positive integer. Furthermore, let $\left\{a_{k}\right\}$ be the sequence of the modified Ganelius sampling points given by Definition 2.5. Then,

$$
\max _{s \in[0,1]} s^{r} \prod_{k=1}^{N}\left|\frac{s-a_{k}}{s+a_{k}}\right| \leq C \exp (-\pi \sqrt{N r})
$$

holds, where $C$ is a positive number independent of $N$.

The sequence $\left\{a_{k}\right\}$ given by Definition 2.5 is contained in the interval $(0,1)$. We need to transform this sequence to that on the interval $(-1,1)$ for the approximation formula on $(-1,1)$. Let $b_{k}$ be defined by

$$
b_{k}:=\sqrt{\frac{1-a_{k}}{1+a_{k}}}, \quad b_{-k}:=-b_{k} \quad(k=1,2, \ldots, N)
$$

and let $\beta_{k}$ be defined by

$$
\beta_{k}:=\tanh \left(\frac{2 d}{\pi} \operatorname{arctanh} b_{k}\right) \quad(k= \pm 1, \ldots, \pm N) .
$$

Both of the sequences $\left\{b_{k}\right\}$ and $\left\{\beta_{k}\right\}$ are contained in $(-1,1)$. Furthermore, we define $\sigma_{k}$ by

$$
\sigma_{k}:=\prod_{\substack{\ell=-N \\ \ell \neq k}}^{N} \frac{1-b_{\ell} b_{k}}{b_{k}-b_{\ell}}
$$

for the coefficients of the formula, where the symbol ' of the product symbol means exclusion of $k=0$. We also use the same symbol for the summation symbol. Finally, in order to construct basis functions for the formula, we define a function $B_{N}(z ; \boldsymbol{\beta}, d)$ by

$$
B_{N}(z ; \boldsymbol{\beta}, d):=\prod_{k=-N}^{N} \tanh \left[\frac{\pi}{2 d}\left(\operatorname{arctanh} z-\operatorname{arctanh} \beta_{k}\right)\right] .
$$

Remark 2.7. After some algebra, we can obtain the expression

$$
B_{N}(z ; \boldsymbol{\beta}, d)=\prod_{k=-N}^{N} \frac{\left[\left(1-\beta_{k}\right)(1+z)\right]^{\pi /(2 d)}-\left[\left(1+\beta_{k}\right)(1-z)\right]^{\pi /(2 d)}}{\left[\left(1-\beta_{k}\right)(1+z)\right]^{\pi /(2 d)}+\left[\left(1+\beta_{k}\right)(1-z)\right]^{\pi /(2 d)}} .
$$

Therefore, if $d=\pi /(2 m)$ for a positive integer $m$, the function $B_{N}(z ; \boldsymbol{\beta}, d)$ is a rational function. In particular, if $d=\pi / 2$, we have $\beta_{k}=b_{k}$ and

$$
B_{N}(z ; \boldsymbol{\beta}, \pi / 2)=\prod_{k=-N}^{N} \frac{z-b_{k}}{1-b_{k} z},
$$

which is known as the Blaschke product. Therefore, the function given by (2.15) is its generalization.

By using the sequences and function defined above, Ushima et al. 11] have proposed the approximation formula $\tilde{f}_{N}$ given by

$$
f(x) \approx \tilde{f}_{N}(x):=\sum_{k=-N}^{N} f\left(\beta_{k}\right) \frac{2 d \sigma_{k}}{\pi} \frac{\left(1-x^{2}\right) B_{N}(x ; \boldsymbol{\beta}, d)}{x-\beta_{k}} .
$$


Then, by using Theorem 2.6 they have given its error estimate as follows.

Theorem 2.8 (11]). Let $\mu$ be a real number satisfying $0<\mu<\min \{2, \pi / d\}$. Then, for $\tilde{f}_{N}$ given by (2.16), we have

$$
\sup _{\substack{f \in \boldsymbol{H}^{\infty}\left(\mathcal{D}_{d}, w_{\mu}\right) \\\|f\| \leq 1}}\left(\sup _{x \in(-1,1)}\left|f(x)-\tilde{f}_{N}(x)\right|\right) \leq C \exp (-\sqrt{\pi d \mu N}),
$$

where $C$ is a positive number independent of $N$.

2.5. Contribution of this paper. In Theorem 2.8 the assumption $\mu<\min \{2, \pi / d\}$ is originated from the form of Formula (2.16) and the assumption $r<1$ in Theorem [2.6. In this paper, by generalizing Formula (2.16), we propose a new approximation formula in $\boldsymbol{H}^{\infty}\left(\mathcal{D}_{d}, w_{\mu}\right)$ for any $\mu>0$ and generalize Theorem 2.6 by removing the assumption $r<1$. Then, we give the error estimate of the new formula by a generalization of Theorem 2.8 in which the assumption $\mu<\min \{2, \pi / d\}$ is removed. As shown below, the generalized versions of Formula (2.16), Theorem 2.6 and Theorem 2.8 are Formula (3.2), Theorem 4.2 and Theorem 3.3, respectively.

\section{An approximation formula by the Ganelius SAMPling Points and GENERALIZED BLASCHKE PRODUCT}

3.1. Main result. For a function $f \in \boldsymbol{H}^{\infty}\left(\Lambda_{d}, w_{\mu}\right)$, by using a real number $\nu$ with

$$
\mu / 2<\nu<\mu / 2+1,
$$

we propose the approximation formula $\tilde{f}_{\nu, N}(x)$ given by

$$
f(x) \approx \tilde{f}_{\nu, N}(x):=\sum_{k=-N}^{N} f\left(\beta_{k}\right) \frac{2 d \sigma_{k}}{\pi} \frac{\left(1-x^{2}\right)^{\nu}}{\left(1-\beta_{k}^{2}\right)^{\nu-1}} \frac{B_{N}(x ; \boldsymbol{\beta}, d)}{x-\beta_{k}} .
$$

Remark 3.1. In the case that $\mu<2$, by choosing $\nu=1$, we can obtain Formula (2.16) from Formula (3.2).

Remark 3.2. According to (3.1), we can set $\nu=\lceil\mu / 2\rceil$ if $\mu$ is not an even integer. From this fact and Remark 2.7 in the case that $\mu$ is not an even integer and $d=\pi /(2 m)$ for a positive integer $m$, the approximant $\tilde{f}_{\nu, N}(x)$ becomes a rational function by letting $\nu=\lceil\mu / 2\rceil$. In such a case, it may be better to use the rational approximant from a practical point of view.

In the following, we give an upper bound of the error of Formula (3.2) and show its optimality by the fact that the upper bound has the same order as the lower bound of $E_{2 N}^{\min }\left(\boldsymbol{H}^{\infty}\left(\Lambda_{d}, w_{\mu}\right)\right)$ given by (2.5). The upper bound is given by the following theorem.

Theorem 3.3. Let $d$ be a positive number satisfying $0<d<\pi$, and let $\mu$ and $\nu$ be positive numbers satisfying (3.1). Then, for $\tilde{f}_{\nu, N}$ given by (3.2), we have

$$
\sup _{\substack{f \in \boldsymbol{H}^{\infty}\left(\Lambda_{d}, w_{\mu}\right) \\\|f\| \leq 1}}\left(\sup _{x \in(-1,1)}\left|f(x)-\tilde{f}_{\nu, N}(x)\right|\right) \leq C \exp (-\sqrt{\pi d \mu N}),
$$

where $C$ is a positive number independent of $N$. 
3.2. Sketch of the proof of Theorem 3.3. Theorem 3.3 follows from Lemmas 3.4 3.6 below, whose proofs are shown in Section 4 . In order to state the lemmas, for a nonnegative real number $\delta$, we define $\Lambda_{d}(\delta)$ by

$$
\Lambda_{d}(\delta):=\Lambda_{d} \cap\left\{z\left|\inf _{\zeta \in \partial \Lambda_{d}}\right| z-\zeta \mid>\delta\right\},
$$

and set the counterclockwise direction to its boundary $\partial \Lambda_{d}(\delta)$.

Lemma 3.4. We have

$$
\begin{aligned}
& \sup _{\substack{f \in \boldsymbol{H}^{\infty}\left(\Lambda_{d}, w_{\mu}\right) \\
\|f\| \leq 1}}\left(\sup _{x \in(-1,1)}\left|f(x)-\tilde{f}_{\nu, N}(x)\right|\right) \\
& \leq \sup _{x \in(-1,1)} \frac{1}{2 \pi}\left(1-x^{2}\right)^{\nu}\left|B_{n}(x ; \boldsymbol{\beta}, d)\right| \lim _{\delta \rightarrow+0} \oint_{\partial \Lambda_{d}(\delta)}\left|\frac{\left(1-z^{2}\right)^{\mu / 2-\nu}}{z-x}\right||\mathrm{d} z| .
\end{aligned}
$$

Lemma 3.5. Let $x$ be a real number with $x \in(-1,1)$. Then, we have

$$
\lim _{\delta \rightarrow+0} \oint_{\partial \Lambda_{d}(\delta)}\left|\frac{\left(1-z^{2}\right)^{\mu / 2-\nu}}{z-x}\right||\mathrm{d} z| \leq C_{1}\left(1-x^{2}\right)^{\mu / 2-\nu} .
$$

where $C_{1}$ is a positive real number independent of $x$.

Lemma 3.6. We have

$$
\sup _{x \in(-1,1)}\left(1-x^{2}\right)^{\mu / 2}\left|B_{N}(x ; \boldsymbol{\beta}, d)\right| \leq C_{2} \exp (-\sqrt{\pi d \mu N}),
$$

where $C_{2}$ is a positive real number independent of $N$.

Then, Theorem 3.3 is proven as follows. We derive from Lemmas 3.4 and 3.5 that

$$
\begin{aligned}
& \sup _{\substack{f \in \boldsymbol{H}^{\infty}\left(\Lambda_{d}, w_{\mu}\right) \\
\|f\| \leq 1}}\left(\sup _{x \in(-1,1)}\left|f(x)-\tilde{f}_{\nu, N}(x)\right|\right) \\
& \leq \sup _{x \in(-1,1)} \frac{C_{1}}{2 \pi}\left(1-x^{2}\right)^{\mu / 2}\left|B_{n}(x ; \boldsymbol{\beta}, d)\right| .
\end{aligned}
$$

Then, by Lemma 3.6 we have the estimate in 3.3) in Theorem 3.3. Finally, by Theorem 2.3 with $n=2 N$ and Theorem 3.3 we have

$$
\begin{aligned}
c \exp (-\sqrt{\pi d \mu N}) & \leq E_{2 N}^{\min }\left(\boldsymbol{H}^{\infty}\left(\Lambda_{d}, w_{\mu}\right)\right) \\
& \leq \sup _{\substack{f \in \boldsymbol{H}^{\infty}\left(\Lambda_{d}, w_{\mu}\right) \\
\|f\| \leq 1}}\left(\sup _{x \in(-1,1)}\left|f(x)-\tilde{f}_{\nu, N}(x)\right|\right) \\
& \leq C \exp (-\sqrt{\pi d \mu N}),
\end{aligned}
$$

which guarantees the optimality of Formula (3.2). 


\section{Proofs of Lemmas 3.43 .6}

4.1. Proof of Lemma 3.4. Let $x \in(-1,1)$ and let $\delta$ be a positive number such that $\{x\} \cup\left\{\beta_{k}\right\} \subset \Lambda_{d}(\delta)$. We begin with writing the difference $f(x)-\tilde{f}_{\nu, N}(x)$ by an complex contour integral. By using $g(z):=f(z) /\left(1-z^{2}\right)^{\nu}$, we define $I_{\Lambda_{d}(\delta)}(x)$ by

$$
I_{\Lambda_{d}(\delta)}(x):=\frac{1}{2 \pi \mathrm{i}} \oint_{\partial \Lambda_{d}(\delta)} \frac{1}{B_{N}(z ; \boldsymbol{\beta}, d)} \frac{g(z)}{z-x} \mathrm{~d} z .
$$

Because the function $g$ is analytic on $\Lambda_{d}(\delta)$ and bounded on the closure of $\Lambda_{d}(\delta)$, it follows from the residue theorem that

$$
I_{\Lambda_{d}(\delta)}(x)=\frac{g(x)}{B_{N}(x ; \boldsymbol{\beta}, d)}-\sum_{k=-N}^{N} g\left(\beta_{k}\right) \frac{2 d \sigma_{k}}{\pi} \frac{1-\beta_{k}^{2}}{x-\beta_{k}} .
$$

By multiplying both sides of the above equality by $\left(1-x^{2}\right)^{\nu} B_{N}(x ; \boldsymbol{\beta}, d)$, we have

$$
\begin{aligned}
& \left(1-x^{2}\right)^{\nu} B_{N}(x ; \boldsymbol{\beta}, d) I_{\Lambda_{d}(\delta)}(x) \\
& =f(x)-\sum_{k=-N}^{N} f\left(\beta_{k}\right) \frac{2 d \sigma_{k}}{\pi} \frac{\left(1-x^{2}\right)^{\nu}}{\left(1-\beta_{k}^{2}\right)^{\nu-1}} \frac{B_{N}(x ; \boldsymbol{\beta}, d)}{x-\beta_{k}} \\
& =f(x)-\tilde{f}_{\nu, N}(x) .
\end{aligned}
$$

Then, we bound $\left|I_{\Lambda_{d}(\delta)}(x)\right|$ from above for $f \in \boldsymbol{H}^{\infty}\left(\Lambda_{d}, w_{\mu}\right)$ with $\|f\| \leq 1$ and $x \in(-1,1)$ as follows:

$$
\begin{aligned}
& \left|I_{\Lambda_{d}(\delta)}(x)\right| \\
& =\frac{1}{2 \pi}\left|\oint_{\partial \Lambda_{d}(\delta)} \frac{1}{B_{N}(z ; \boldsymbol{\beta}, d)} \frac{f(z)}{\left(1-z^{2}\right)^{\mu / 2}} \frac{\left(1-z^{2}\right)^{\mu / 2-\nu}}{z-x} \mathrm{~d} z\right| \\
& \leq \frac{1}{2 \pi} \max _{z \in \partial \Lambda_{d}(\delta)}\left|\frac{1}{B_{N}(z ; \boldsymbol{\beta}, d)}\right| \oint_{\partial \Lambda_{d}(\delta)}\left|\frac{\left(1-z^{2}\right)^{\mu / 2-\nu}}{z-x}\right||\mathrm{d} z| .
\end{aligned}
$$

Finally, we can derive the conclusion of Lemma 3.4 from (4.2), (4.3), and the fact that

$$
\lim _{\delta \rightarrow+0} \max _{z \in \partial \Lambda_{d}(\delta)}\left|\frac{1}{B_{N}(z ; \boldsymbol{\beta}, d)}\right|=1 .
$$

This equality follows from the expression

$$
\begin{aligned}
B_{N}(z ; \boldsymbol{\beta}, d) & =\prod_{k=-N}^{N} \tanh \left[\frac{\pi}{2 d}\left(\zeta / 2-\operatorname{arctanh} \beta_{k}\right)\right] \\
& =\prod_{k=-N}^{N} \tanh \left[\frac{\pi}{2 d}\left(\operatorname{Re} \zeta / 2-\operatorname{arctanh} \beta_{k}\right)+\frac{\pi}{4 d}(\operatorname{Im} \zeta) \mathrm{i}\right],
\end{aligned}
$$

where $\zeta=2 \operatorname{arctanh} z \in \mathcal{D}_{d}$ (see (2.1) $)$. Note that $z \in \partial \Lambda_{d} \Longleftrightarrow|\operatorname{Im} \zeta|=d$ and $|\tanh [s \pm(\pi / 4) \mathrm{i}]|=1$ for any $s \in \mathbf{R}$. 
4.2. Proof of Lemma 3.5. It suffices to bound

$$
I(d, \mu, \nu ; x):=\oint_{\partial \Lambda_{d}}\left|\frac{\left(1-z^{2}\right)^{\mu / 2-\nu}}{z-x}\right||\mathrm{d} z|
$$

from above by the RHS of (3.6). Owing to the symmetry of the contour $\partial \Lambda_{d}$ and the integrand with respect to the real axis, we only consider its upper half. We employ the variable transformations given by

$$
z=\tanh \left(\frac{s+d \mathrm{i}}{2}\right) \quad(-\infty<s<\infty)
$$

and $x=\tanh (t / 2)$ with $t \in \mathbf{R}$ to obtain

$$
\begin{aligned}
& \frac{1}{2} I(d, \mu, \nu ; \tanh (t / 2)) \\
& =\frac{1}{2} \int_{-\infty}^{\infty} \frac{1}{|\tanh ((s+d \mathrm{i}) / 2)-\tanh (t / 2)|} \frac{1}{|\cosh ((s+d \mathrm{i}) / 2)|^{\mu-2 \nu+2}} \mathrm{~d} s .
\end{aligned}
$$

Because we have

$$
\begin{aligned}
& |\tanh ((s+d \mathrm{i}) / 2)-\tanh (t / 2)|^{2}=\frac{1}{\cosh ^{2}(t / 2)} \frac{\cosh (s-t)-\cos d}{\cosh s+\cos d}, \\
& |\cosh ((s+d \mathrm{i}) / 2)|^{2}=\frac{1}{2}(\cosh s+\cos d)
\end{aligned}
$$

after some algebra, it follows from (4.4), (4.5), and (4.6) that

$$
\begin{aligned}
& I(d, \mu, \nu ; \tanh (t / 2)) \\
& =\int_{-\infty}^{\infty} \frac{2^{(\mu-2 \nu+2) / 2} \cosh (t / 2)}{(\cosh (s-t)-\cos d)^{1 / 2}(\cosh s+\cos d)^{(\mu-2 \nu+1) / 2}} \mathrm{~d} s .
\end{aligned}
$$

Then, by noting that $|\cos d|<1$ and that

$$
(1-|\cos d|) \cosh u \leq \cosh u \pm \cos d \leq(1+|\cos d|) \cosh u
$$

holds for a real number $u$, we can derive from (4.7) that

$$
I(d, \mu, \nu ; \tanh (t / 2)) \leq U_{d, \mu, \nu} \cosh (t / 2) J(1 / 2,(\mu-2 \nu+1) / 2 ; t),
$$

where

$$
U_{d, \mu, \nu}:=2^{(\mu-2 \nu+2) / 2} \max \left\{\frac{1}{(1-|\cos d|)^{(\mu-2 \nu+2) / 2}}, \frac{(1+|\cos d|)^{(2 \nu-1-\mu) / 2}}{(1-|\cos d|)^{1 / 2}}\right\},
$$

and

$$
J(\alpha, \beta ; t):=\int_{-\infty}^{\infty} \frac{1}{\cosh ^{\alpha}(s-t) \cosh ^{\beta} s} \mathrm{~d} s .
$$

Therefore, what remains is to estimate $J(\alpha, \beta ; t)$ given by (4.9). This estimate is done as shown in the following lemma also used in [11. For readers' convenience, we describe its proof in Appendix $\mathrm{A}$

Lemma 4.1 ([11, Lemma 3.4]). Let $\alpha$ and $\beta$ be distinct real numbers with $\alpha+\beta>0$ and let $a=\max \{\alpha, \beta\}$ and $b=\min \{\alpha, \beta\}$. Then, for a real number $t$, we have

$$
J(\alpha, \beta ; t) \leq \frac{\max \left\{2^{a+1}, 2^{a+b+1}\right\}}{a^{2}-b^{2}}\left(-b \mathrm{e}^{-a|t|}+a \mathrm{e}^{-b|t|}\right) .
$$


By Lemma 4.1 we have

$$
J(1 / 2,(\mu-2 \nu+1) / 2 ; t) \leq \tilde{C}_{\mu, \nu}\left\{(2 \nu-1-\mu) \mathrm{e}^{-|t| / 2}+\mathrm{e}^{-((\mu-2 \nu+1) / 2)|t|}\right\},
$$

where $\tilde{C}_{\mu, \nu}$ is a positive number depending only on $\mu$ and $\nu$. Therefore, it follows from (4.8) and (4.11) that

$$
I(d, \mu, \nu ; \tanh (t / 2)) \leq \hat{C}_{d, \mu, \nu}\left\{\cosh ^{2}(t / 2)\right\}^{-(\mu / 2-\nu)},
$$

where $\hat{C}_{d, \mu, \nu}$ is a positive number depending only on $d, \mu$, and $\nu$. By converting Inequality (4.12) into that with respect to $x$ by the transform $t=2 \operatorname{arctanh} x=$ $\log ((1+x) /(1-x))$, we obtain the concluding inequality in Lemma 3.5.

4.3. Proof of Lemma 3.6. We reduce Lemma 3.6 to the following theorem, which is a generalization of Theorem [2.6 ([3, Lemma 1]). We prove this theorem in Appendix B.

Theorem 4.2. Let $r$ be a positive real number and let $N$ be a positive integer. Furthermore, let $\left\{a_{k}\right\}$ be the sequence of the modified Ganelius sampling points given by Definition 2.5. Then,

$$
\max _{s \in[0,1]} s^{r} \prod_{k=1}^{N}\left|\frac{s-a_{k}}{s+a_{k}}\right| \leq C \exp (-\pi \sqrt{N r})
$$

holds, where $C$ is a positive number independent of $N$.

Remark 4.3. We just assume that $r>0$ in Theorem 4.2 as opposed to Theorem 2.6.

As stated right after Lemma 1 in [3], owing to the variable transformation

$$
s=\frac{1-t^{2}}{1+t^{2}}
$$

Inequality (4.13) in Theorem 4.2 is equivalent to

$$
\max _{t \in[-1,1]}\left(1-t^{2}\right)^{r} \prod_{k=-N}^{N}\left|\frac{t-b_{k}}{1-b_{k} t}\right| \leq C_{3}^{\prime} \exp (-\pi \sqrt{N r}),
$$

where $C_{3}^{\prime}$ is a positive number independent of $N$. In the following, we reduce Inequality (3.7) in Lemma 3.6 to Inequality (4.15).

First, by letting

$$
x=\tanh \left(\frac{2 d}{\pi} \operatorname{arctanh} t\right)
$$

for $t \in[-1,1]$, we have

$$
B_{N}(x ; \boldsymbol{\beta}, d)=\prod_{k=-N}^{N} \frac{t-b_{k}}{1-b_{k} t} .
$$

Next, by noting that $\operatorname{arctanh} t=(1 / 2) \log ((1+t) /(1-t))$, we have

$$
\begin{aligned}
1-x^{2} & =\frac{1}{\cosh ^{2}((2 d / \pi) \operatorname{arctanh} t)} \\
& =\left(\frac{2\left(1-t^{2}\right)^{d / \pi}}{(1+t)^{2 d / \pi}+(1-t)^{2 d / \pi}}\right)^{2} \\
& \leq 2^{2(2 d / \pi+1)}\left(1-t^{2}\right)^{2 d / \pi}
\end{aligned}
$$


where we employ the inequality $A^{\alpha}+B^{\alpha} \geq[(A+B) / 2]^{\alpha}$ that holds for any positive numbers $\alpha, A$, and $B$. From these and (4.15), by letting $r=d \mu / \pi$, we have

$$
\begin{aligned}
\left(1-x^{2}\right)^{\mu / 2}\left|B_{N}(x ; \boldsymbol{\beta}, d)\right| & \leq 2^{(2 d / \pi+1) \mu}\left(1-t^{2}\right)^{d \mu / \pi} \prod_{k=-N}^{N}\left|\frac{t-b_{k}}{1-b_{k} t}\right| \\
& \leq C_{3}^{\prime \prime} \exp (-\sqrt{\pi d \mu N})
\end{aligned}
$$

where $C_{3}^{\prime \prime}$ is a positive number independent of $N$. Thus Lemma 3.6 is proven.

\section{Numerical EXPERIMENTS}

In this section, we compute the approximations of some functions by Formula (3.2) and observe their errors. Moreover, we compare the errors with those of the SE-Sinc approximations given by Formula (2.6) for the same functions.

We adopt the following functions for this numerical experiment.

$$
\begin{array}{ll}
\text { Example } 1 & f_{1}(x):=\sqrt{\frac{1-x^{2}}{1+x^{2}}} \\
\text { Example } 2 & f_{2}(x):=\sqrt{\frac{3-3 x^{2}}{1+3 x^{2}}} \\
\text { Example } 3 & f_{3}(x):=\sqrt{\frac{1-x^{2}}{3+x^{2}}} \\
\text { Example } 4 & f_{4}(x):=\left(1-x^{2}\right)^{1 / \sqrt{2}} \sqrt{\cos (4 \operatorname{arctanh} x)+\cosh \pi} \\
\text { Example } 5 & f_{5}(x):=\left(\frac{1-x^{2}}{1+x^{2}}\right)^{3 / 2} .
\end{array}
$$

These functions have the singularities at $x= \pm 1$. Table 1 shows the other singularities of $f_{1}, \ldots, f_{5}$ and the parameters $d$ and $\mu$ such that $f_{i} \in \boldsymbol{H}^{\infty}\left(\mathcal{D}_{d}, w_{\mu}\right)$ for $i=1, \ldots, 5$. We can adopt arbitrary positive values of $\varepsilon_{1}, \varepsilon_{2}, \varepsilon_{3}$, and $\varepsilon_{5}$ as long as

\begin{tabular}{|c|c|c|c|}
\hline & Singularities & $d$ & $\mu$ \\
\hline$f_{1}$ & $\pm \mathrm{i}$ & $\pi / 2-\varepsilon_{1}$ & 1 \\
\hline$f_{2}$ & $\pm \mathrm{i} / \sqrt{3}$ & $\pi / 3-\varepsilon_{2}$ & 1 \\
\hline$f_{3}$ & $\pm \mathrm{i} \sqrt{3}$ & $2 \pi / 3-\varepsilon_{3}$ & 1 \\
\hline$f_{4}$ & $\tanh [m+(1 \pm \mathrm{i}) \pi / 2) / 2] \quad(m \in \mathbf{Z})$ & $\pi / 2$ & $\sqrt{2}$ \\
\hline$f_{5}$ & & $\pi / 2-\varepsilon_{5}$ & 3 \\
\hline
\end{tabular}
$d>0$ in Table1. In this experiment, we set $\varepsilon_{1}=\varepsilon_{5}=\pi / 2-1.57, \varepsilon_{2}=\pi / 3-1.047$, and $\varepsilon_{3}=2 \pi / 3-2.094$. The function $f_{5}$ is an example that does not satisfy the old condition $\mu<\min \{2, \pi / d\}$ assumed in Theorem 2.8 .

TABLE 1. The singularities other than \pm 1 and parameters $d$ and $\mu$ of $f_{1}, \ldots, f_{5}$. The positive numbers $\varepsilon_{1}, \varepsilon_{2}, \varepsilon_{3}$, and $\varepsilon_{5}$ are arbitrary as long as $d>0$.

In order to use Formula (3.2), we need to decide the value of $\nu$ in $\tilde{f}_{\nu, N}$. We set $\nu=\lceil\mu / 2\rceil$, i.e., $\nu=1$ for $f_{1}, \ldots, f_{4}$ and $\nu=2$ for $f_{5}$. Then, for a fixed $N$ and 
each of the functions $f=f_{1}, \ldots, f_{5}$, we compute the values of $f(x)-\tilde{f}_{\nu, N}(x)$ for $x \in X \cup Y \subset(-1,1)$, where

$$
\begin{aligned}
& X:=\{i / 1000 \mid i=-999, \ldots, 999\}, \\
& Y:=\left\{ \pm\left(1-k / 10^{\ell}\right) \mid \ell=4, \ldots, 16, k=1, \ldots, 9\right\},
\end{aligned}
$$

and adopt the maximum of their absolute values as the computed error. Because the computed error is often attained at the points close to the endpoints \pm 1 , we employ the set $Y$ in order to capture those points. For all the computations, we used a computer with PowerPC G5 Dual 2 GHz CPU and GCC 4.0.1 compiler, and programs written in $\mathrm{C}$ with all the floating point numbers declared as the "long double" variables. Then, all the computations are done with the quadruple precision floating point numbers.

We show the computed errors for $N=4,9,16, \ldots, 144$ in Figures 2 6 , In each figure, the legends "SE-Sinc" and "Ganelius" indicate the results of Formulas (2.6) and (3.2), respectively. Note that the total number $n$ of the sampling points is $n=2 N+1$ for Formula (2.6) and $n=2 N$ for Formula (3.2). Furthermore, we estimate the decay rate of the errors by computing the ratio (the error for $\left.N=(m-1)^{2}\right) /\left(\right.$ the error for $N=m^{2}$ ) for $m=2,3,4, \ldots, 12$. The theoretical values of the ratio for Formulas (2.6) and (3.2) are $\exp (\sqrt{\pi d \mu / 2})$ and $\exp (\sqrt{\pi d \mu})$, respectively. We show the computed ratios ("rate") and theoretical values ("t.v.") in Tables 26 .

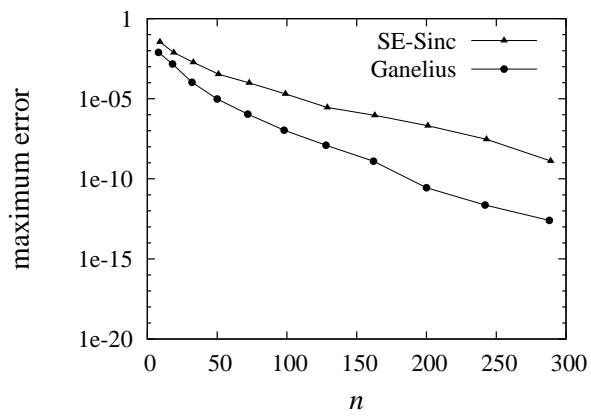

TABLE 2. Convergence rate of the approximation of $f_{1}$.

\begin{tabular}{rlrlr}
\hline$N$ & Ganelius & rate & SE-Sinc & rate \\
\hline 4 & $7.73 \mathrm{E}^{-3}$ & & $3.48 \mathrm{E}^{-2}$ & \\
9 & $1.47 \mathrm{E}^{-3}$ & 5.2 & $7.49 \mathrm{E}^{-3}$ & 4.6 \\
16 & $1.06 \mathrm{E}^{-4}$ & 13.8 & $1.88 \mathrm{E}^{-3}$ & 3.9 \\
25 & $9.57 \mathrm{E}^{-6}$ & 11.0 & $3.38 \mathrm{E}^{-4}$ & 5.5 \\
36 & $1.10 \mathrm{E}^{-6}$ & 8.6 & $9.67 \mathrm{E}^{-5}$ & 3.5 \\
49 & $1.07 \mathrm{E}^{-7}$ & 10.2 & $1.98 \mathrm{E}^{-5}$ & 4.8 \\
64 & $1.25 \mathrm{E}^{-8}$ & 8.5 & $2.85 \mathrm{E}^{-6}$ & 6.9 \\
81 & $1.25 \mathrm{E}^{-9}$ & 9.9 & $9.23 \mathrm{E}^{-7}$ & 3.0 \\
100 & $2.78 \mathrm{E}^{-11}$ & 45.1 & $2.04 \mathrm{E}^{-7}$ & 4.5 \\
121 & $2.31 \mathrm{E}^{-12}$ & 12.0 & $2.92 \mathrm{E}^{-8}$ & 6.9 \\
144 & $2.55 \mathrm{E}^{-13}$ & 9.0 & $1.30 \mathrm{E}^{-9}$ & 22.3 \\
t.v. & & 9.2 & & 4.8 \\
\hline
\end{tabular}

From these results, we can observe that in each case Formula (3.2) outperforms Formula (2.6) and the sequence of the computed values of the "rate" approaches its theoretical value as $N$ increases.

\section{Concluding Remarks}

In this paper, we proposed the approximation formula given by (3.2) that is optimal in the space $\boldsymbol{H}^{\infty}\left(\mathcal{D}_{d}, w_{\mu}\right)$ for any $\mu>0$. Formula (3.2) is a generalization of Formula (2.16) proposed by Ushima et al. 11, which is valid only in the case that $\mu<\min \{2, \pi / d\}$. In order to estimate the error of Formula (3.2), we showed 


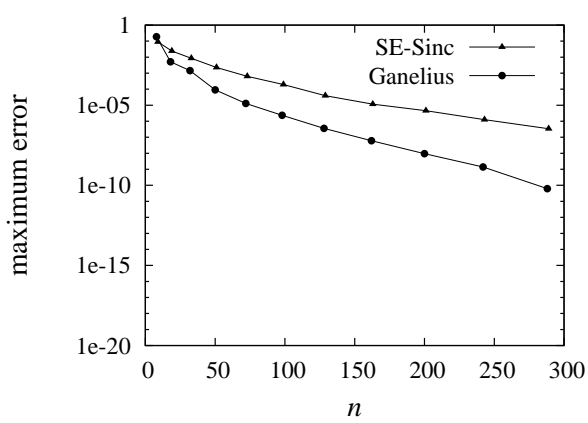

FiguRE 3. Maximum error in the approximation of $f_{2}$.
TABle 3. Convergence rate of in the approximation of $f_{2}$.

\begin{tabular}{rlrll}
\hline$N$ & Ganelius & rate & SE-Sinc & rate \\
\hline 4 & $1.89 \mathrm{E}^{-1}$ & & $8.96 \mathrm{E}^{-2}$ & \\
9 & $5.17 \mathrm{E}^{-3}$ & 36.5 & $2.40 \mathrm{E}^{-2}$ & 3.7 \\
16 & $1.44 \mathrm{E}^{-3}$ & 3.5 & $8.56 \mathrm{E}^{-3}$ & 2.8 \\
25 & $9.13 \mathrm{E}^{-5}$ & 15.8 & $2.27 \mathrm{E}^{-3}$ & 3.7 \\
36 & $1.28 \mathrm{E}^{-5}$ & 7.1 & $6.41 \mathrm{E}^{-4}$ & 3.5 \\
49 & $2.34 \mathrm{E}^{-6}$ & 5.4 & $1.94 \mathrm{E}^{-4}$ & 3.3 \\
64 & $3.57 \mathrm{E}^{-7}$ & 6.5 & $3.91 \mathrm{E}^{-5}$ & 4.9 \\
81 & $6.06 \mathrm{E}^{-8}$ & 5.8 & $1.15 \mathrm{E}^{-5}$ & 3.3 \\
100 & $9.46 \mathrm{E}^{-9}$ & 6.4 & $4.58 \mathrm{E}^{-6}$ & 2.5 \\
121 & $1.40 \mathrm{E}^{-9}$ & 6.7 & $1.25 \mathrm{E}^{-6}$ & 3.6 \\
144 & $6.17 \mathrm{E}^{-11}$ & 22.7 & $3.39 \mathrm{E}^{-7}$ & 3.6 \\
t.v. & & 6.1 & & 3.6 \\
\hline
\end{tabular}

TABle 4. Convergence rate of in the approximation of $f_{3}$.

\begin{tabular}{rlrlr}
\hline$N$ & Ganelius & rate & SE-Sinc & rate \\
\hline 4 & $3.63 \mathrm{E}^{-3}$ & & $1.33 \mathrm{E}^{-2}$ & \\
9 & $4.35 \mathrm{E}^{-4}$ & 8.3 & $2.33 \mathrm{E}^{-3}$ & 5.7 \\
16 & $2.36 \mathrm{E}^{-5}$ & 18.3 & $5.06 \mathrm{E}^{-4}$ & 4.6 \\
25 & $1.85 \mathrm{E}^{-6}$ & 12.7 & $8.04 \mathrm{E}^{-5}$ & 6.2 \\
36 & $1.22 \mathrm{E}^{-7}$ & 15.1 & $1.52 \mathrm{E}^{-5}$ & 5.2 \\
49 & $1.00 \mathrm{E}^{-8}$ & 12.2 & $2.49 \mathrm{E}^{-6}$ & 6.1 \\
64 & $7.97 \mathrm{E}^{-10}$ & 12.5 & $4.25 \mathrm{E}^{-7}$ & 5.8 \\
81 & $5.76 \mathrm{E}^{-12}$ & 138.4 & $7.14 \mathrm{E}^{-8}$ & 5.9 \\
100 & $3.60 \mathrm{E}^{-13}$ & 15.9 & $1.17 \mathrm{E}^{-8}$ & 6.0 \\
121 & $2.33 \mathrm{E}^{-14}$ & 15.4 & $2.82 \mathrm{E}^{-10}$ & 41.8 \\
144 & $1.83 \mathrm{E}^{-15}$ & 12.7 & $4.39 \mathrm{E}^{-11}$ & 6.4 \\
t.v. & & 13.0 & & 6.1 \\
\hline & & & &
\end{tabular}

Theorem 4.2, a generalization of Theorem 2.6 (the Ganelius theorem [3, Lemma 1]). Then, we gave an upper bound of the error of Formula (3.2) in Theorem 3.3 and showed the optimality of the formula by combining this upper bound and the lower bound of the minimum error norm given by Theorem [2.3 (9. (a) on page 782]). Furthermore, we observed that Formula (3.2) achieved the optimal convergence rate in the numerical experiment.

We can list some themes for future work about the optimal approximation in $\boldsymbol{H}^{\infty}\left(\Lambda_{d}, w_{\mu}\right)$ : finding other optimal formulas and comparing them with Formula (3.2), inventing improved methods for fast computation by Formula (3.2), applying Formula (3.2) to differential equations such as two point boundary problems, etc. 


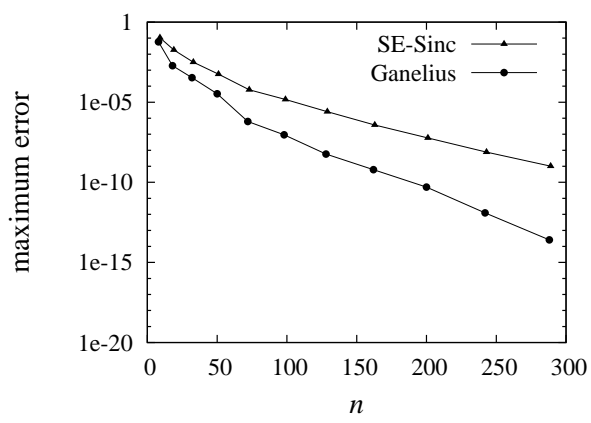

TABle 5. Convergence rate of the approximation of $f_{4}$.

\begin{tabular}{rlrll}
\hline$N$ & Ganelius & rate & SE-Sinc & rate \\
\hline 4 & $5.83 \mathrm{E}^{-2}$ & & $1.06 \mathrm{E}^{-1}$ & \\
9 & $1.90 \mathrm{E}^{-3}$ & 30.6 & $1.81 \mathrm{E}^{-2}$ & 5.8 \\
16 & $3.41 \mathrm{E}^{-4}$ & 5.5 & $3.14 \mathrm{E}^{-3}$ & 5.7 \\
25 & $3.35 \mathrm{E}^{-5}$ & 10.1 & $5.59 \mathrm{E}^{-4}$ & 5.6 \\
36 & $6.26 \mathrm{E}^{-7}$ & 53.6 & $5.95 \mathrm{E}^{-5}$ & 9.3 \\
49 & $9.30 \mathrm{E}^{-8}$ & 6.7 & $1.47 \mathrm{E}^{-5}$ & 4.0 \\
64 & $5.77 \mathrm{E}^{-9}$ & 16.0 & $2.54 \mathrm{E}^{-6}$ & 5.7 \\
81 & $6.14 \mathrm{E}^{-10}$ & 9.3 & $3.78 \mathrm{E}^{-7}$ & 6.7 \\
100 & $5.04 \mathrm{E}^{-11}$ & 12.1 & $5.88 \mathrm{E}^{-8}$ & 6.4 \\
121 & $1.23 \mathrm{E}^{-12}$ & 40.9 & $7.63 \mathrm{E}^{-9}$ & 7.7 \\
144 & $2.55 \mathrm{E}^{-14}$ & 48.1 & $1.01 \mathrm{E}^{-9}$ & 7.5 \\
t.v. & & 14.0 & & 6.5 \\
\hline
\end{tabular}

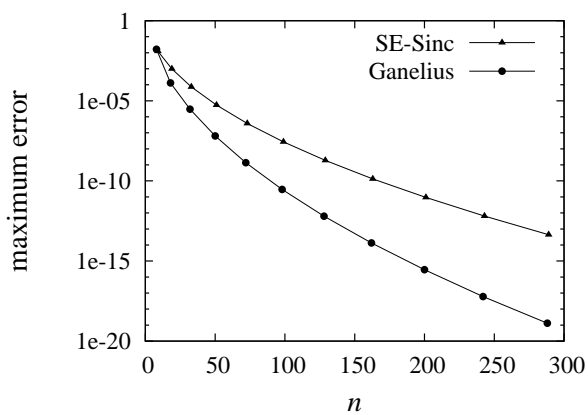

TABle 6. Convergence rate of the approximation of $f_{5}$.

\begin{tabular}{rlrll}
\hline$N$ & Ganelius & rate & SE-Sinc & rate \\
\hline 4 & $1.64 \mathrm{E}^{-2}$ & & $1.24 \mathrm{E}^{-2}$ & \\
9 & $1.30 \mathrm{E}^{-4}$ & 126.4 & $9.91 \mathrm{E}^{-4}$ & 12.5 \\
16 & $2.98 \mathrm{E}^{-6}$ & 43.6 & $7.37 \mathrm{E}^{-5}$ & 13.4 \\
25 & $6.43 \mathrm{E}^{-8}$ & 46.4 & $5.38 \mathrm{E}^{-6}$ & 13.6 \\
36 & $1.38 \mathrm{E}^{-9}$ & 46.6 & $3.85 \mathrm{E}^{-7}$ & 13.9 \\
49 & $2.93 \mathrm{E}^{-11}$ & 46.9 & $2.72 \mathrm{E}^{-8}$ & 14.1 \\
64 & $6.29 \mathrm{E}^{-13}$ & 46.6 & $1.91 \mathrm{E}^{-9}$ & 14.2 \\
81 & $1.33 \mathrm{E}^{-14}$ & 47.0 & $1.33 \mathrm{E}^{-10}$ & 14.3 \\
100 & $2.85 \mathrm{E}^{-16}$ & 46.9 & $9.23 \mathrm{E}^{-12}$ & 14.4 \\
121 & $6.06 \mathrm{E}^{-18}$ & 46.9 & $6.36 \mathrm{E}^{-13}$ & 14.5 \\
144 & $1.30 \mathrm{E}^{-19}$ & 46.4 & $4.36 \mathrm{E}^{-14}$ & 14.5 \\
t.v. & & 46.8 & & 15.1 \\
\hline & & & &
\end{tabular}

Appendix A. Proof of Lemma 4.1

Without loss of generality, we can assume that $t \geq 0, a=\alpha$, and $b=\beta$. The assumptions about $a$ and $b$ are owing to the fact that

$$
\begin{aligned}
J(\alpha, \beta ; t) & =\int_{-\infty}^{\infty} \frac{1}{\cosh ^{\alpha}(t-s) \cosh ^{\beta} s} \mathrm{~d} s \\
& =\int_{-\infty}^{\infty} \frac{1}{\cosh ^{\alpha} u \cosh ^{\beta}(t-u)} \mathrm{d} u=J(\beta, \alpha ; t) .
\end{aligned}
$$

For the proof of this lemma, we employ the following inequalities:

$$
\begin{cases}\mathrm{e}^{u} / 2 \leq \cosh u \leq \mathrm{e}^{u} & (u \geq 0) \\ \mathrm{e}^{-u} / 2 \leq \cosh u \leq \mathrm{e}^{-u} & (u<0) .\end{cases}
$$

In the following, we deal with two cases: (i) $b<0$ and (ii) $b \geq 0$. 
Case (i)

It holds that $a>0>b$ in this case. By using Inequalities A.1 , we have

$$
\frac{(\cosh s)^{-b}}{\cosh ^{a}(s-t)} \leq \begin{cases}2^{a} \mathrm{e}^{-a t} \mathrm{e}^{(a+b) s} & (s<0), \\ 2^{a} \mathrm{e}^{-a t} \mathrm{e}^{(a-b) s} & (0 \leq s \leq t), \\ 2^{a} \mathrm{e}^{a t} \mathrm{e}^{-(a+b) s} & (t<s) .\end{cases}
$$

Therefore, $J(a, b ; t)$ is bounded from above as follows:

$$
\begin{aligned}
J(a, b ; t) & =\left(\int_{-\infty}^{0}+\int_{0}^{t}+\int_{t}^{\infty}\right) \frac{(\cosh s)^{-b}}{\cosh ^{a}(s-t)} \mathrm{d} s \\
& \leq 2^{b}\left[\mathrm{e}^{-a t}\left\{\frac{1}{a+b}+\frac{1}{a-b}\left(\mathrm{e}^{(a-b) t}-1\right)\right\}+\frac{1}{a+b} \mathrm{e}^{-b t}\right] \\
& =\frac{2^{a+1}}{a^{2}-b^{2}}\left(-b \mathrm{e}^{-a t}+a \mathrm{e}^{-b t}\right) .
\end{aligned}
$$

Case (ii)

It holds that $a \geq b \geq 0$ in this case. By using Inequalities (A.1), we have

$$
\frac{1}{\cosh ^{a}(s-t) \cosh ^{b} s} \leq \begin{cases}2^{a+b} \mathrm{e}^{-a t} \mathrm{e}^{(a+b) s} & (s<0), \\ 2^{a+b} \mathrm{e}^{-a t} \mathrm{e}^{(a-b) s} & (0 \leq s \leq t), \\ 2^{a+b} \mathrm{e}^{a t} \mathrm{e}^{-(a+b) s} & (t<s) .\end{cases}
$$

Therefore, in the same manner as Case (i), $J(a, b ; t)$ is bounded from above as follows:

$$
\begin{aligned}
J(a, b ; t) & =\left(\int_{-\infty}^{0}+\int_{0}^{t}+\int_{t}^{\infty}\right) \frac{1}{\cosh ^{a}(s-t) \cosh ^{b} s} \mathrm{~d} s \\
& \leq \frac{2^{a+b+1}}{a^{2}-b^{2}}\left(-b \mathrm{e}^{-a t}+a \mathrm{e}^{-b t}\right) .
\end{aligned}
$$

From the estimates in Cases (i) and (ii), Lemma 4.1 is proven.

\section{Appendix B. Proof of Theorem 4.2}

It is sufficient to consider the case that $N$ is larger than a certain positive constant. Therefore, we assume that $N$ is large enough such that $N_{0}>3$. The conclusion of Theorem 4.2 is equivalent to the statement that

$$
\sum_{k=1}^{N} \log \left|\frac{s+a_{k}}{s-a_{k}}\right| \geq \pi \sqrt{N r}+r \log s+C^{\prime}
$$

holds for any $s \in[0,1]$, where $C^{\prime}$ is a real number independent of $N$ and $s$. We show this statement by proving the following three lemmas.

Lemma B.1. On the same assumption as Theorem 4.2. for all $s \in[0,1]$, we have

$$
\sum_{k=N_{0}+2}^{N} \log \left|\frac{s+a_{k}}{s-a_{k}}\right| \geq \frac{\pi \sqrt{N_{0} r}}{2} s .
$$


Lemma B.2. On the same assumption as Theorem 4.2. for all $s \in[0,1]$, we have

$$
\int_{1 / \varphi\left(N_{0}\right)}^{1} \log \left|\frac{s+t}{s-t}\right| \frac{2 r \log \left(t \varphi\left(N_{0}\right)\right)}{\pi^{2} t} \mathrm{~d} t \geq \pi \sqrt{N_{0} r}+r \log s-\frac{\pi \sqrt{N_{0} r}}{2} s+C^{\prime \prime}
$$

where $C^{\prime \prime}$ is a real number independent of $N_{0}$ and $s$.

Lemma B.3. On the same assumption as Theorem 4.2. for all $s \in[0,1]$, we have

$$
\sum_{k=1}^{N_{0}+1} \log \left|\frac{s+a_{k}}{s-a_{k}}\right|-\int_{1 / \varphi\left(N_{0}\right)}^{1} \log \left|\frac{s+t}{s-t}\right| \frac{2 r \log \left(t \varphi\left(N_{0}\right)\right)}{\pi^{2} t} \mathrm{~d} t \geq C^{\prime \prime \prime},
$$

where $C^{\prime \prime \prime}$ is a real number independent of $N_{0}$ and $s$.

If Lemmas B.1 B.3 are proven, by adding Inequalities (B.2)-(B.4), we have

$$
\sum_{k=1}^{N} \log \left|\frac{s+a_{k}}{s-a_{k}}\right| \geq \pi \sqrt{N_{0} r}+r \log s+\left(C^{\prime \prime}+C^{\prime \prime \prime}\right) .
$$

Furthermore, we have the inequality

$$
\pi \sqrt{N_{0} r} \geq \pi \sqrt{N r}-c
$$

where $c$ is a real number independent of $N$. By using these inequalities, we can obtain Inequality (B.1).

Lemmas B.1 and B.2 have already been proven by Jang \& Haber 3 in the proof on page 221 of [3] and "Proof of (2)" on pages 218-219 of [3], respectively, without using the assumption $r<1$. On the other hand, Inequality (B.4) in Lemma B.3 corresponds to Inequality (3) on page 218 of 3 , where the assumption $r<1$ is employed. Therefore, we need to prove Lemma B.3 by ourselves in order to remove this assumption.

B.1. Proof of Lemma B.3. For $s=0$, the LHS of (B.4) is zero. Furthermore, for $s=a_{k}$, the first term in the LHS is infinity. Therefore, we assume that $s \in$ $(0,1] \backslash\left\{a_{k}\right\}$ in the rest of this section. By letting

$$
g(s, t):=\log \left|\frac{s+t}{s-t}\right|
$$

we have

$$
\log \left|\frac{s+a_{k}}{s-a_{k}}\right|= \begin{cases}g\left(s \varphi\left(N_{0}\right), \varphi(k-1)\right) & \left(k=1,2, \ldots, N_{0}\right), \\ g\left(s \varphi\left(N_{0}\right), \varphi(k-3 / 2)\right) & \left(k=N_{0}+1\right),\end{cases}
$$

and

$$
\int_{1 / \varphi\left(N_{0}\right)}^{1} \log \left|\frac{s+t}{s-t}\right| \frac{2 r \log \left(t \varphi\left(N_{0}\right)\right)}{\pi^{2} t} \mathrm{~d} t=\int_{0}^{N_{0}} g\left(s \varphi\left(N_{0}\right), \varphi(u)\right) \mathrm{d} u,
$$

where the variable transformation $t=\varphi(u) / \varphi\left(N_{0}\right)$ is employed. Furthermore, by letting

$$
c_{r}:=\frac{\pi}{2 \sqrt{r}} \quad \text { and } \quad \eta:=\frac{1}{2 c_{r}} \log \left(s \varphi\left(N_{0}\right)\right),
$$

we have

$$
g\left(s \varphi\left(N_{0}\right), \varphi(u)\right)=g\left(\exp \left(2 c_{r} \eta\right), \exp \left(2 c_{r} \sqrt{u}\right)\right)
$$




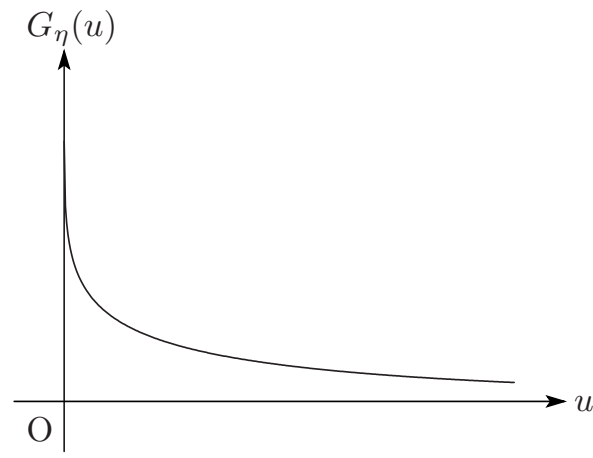

(a) $\eta<0$

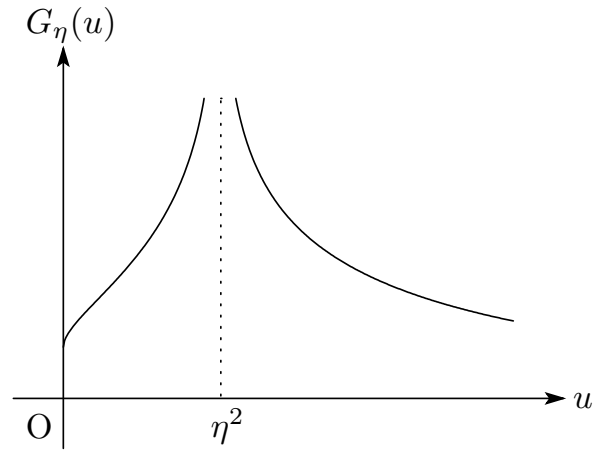

(b) $\eta>0$

Figure 7. Graphs of the function $G_{\eta}$.

$$
=\log \left|\frac{1+\exp \left[2 c_{r}(\sqrt{u}-\eta)\right]}{1-\exp \left[2 c_{r}(\sqrt{u}-\eta)\right]}\right|=-\log \left|\tanh \left[c_{r}(\sqrt{u}-\eta)\right]\right| .
$$

Note that $\eta$ in (B.5) satisfies $-\infty<\eta \leq \sqrt{N_{0}}$ and does not equal the square root of any nonnegative integer because $s \in(0,1] \backslash\left\{a_{k}\right\}$. From these, by letting

$$
G_{\eta}(u):=-\log \left|\tanh \left[c_{r}(\sqrt{u}-\eta)\right]\right|,
$$

we have

$$
\begin{aligned}
& \sum_{k=1}^{N_{0}+1} \log \left|\frac{s+a_{k}}{s-a_{k}}\right|-\int_{1 / \varphi\left(N_{0}\right)}^{1} \log \left|\frac{s+t}{s-t}\right| \frac{2 r \log \left(t \varphi\left(N_{0}\right)\right)}{\pi^{2} t} \mathrm{~d} t \\
& =\sum_{\ell=0}^{N_{0}-1} G_{\eta}(\ell)+G_{\eta}\left(N_{0}-1 / 2\right)-\int_{0}^{N_{0}} G_{\eta}(u) \mathrm{d} u .
\end{aligned}
$$

In the following, we prove the RHS of (B.7) is bounded from below for $\eta$ with $-\infty<\eta \leq \sqrt{N_{0}}$, which completes the proof of Lemma B.3 Note that, as shown by Figure 7 , the function $G_{\eta}(u)$ in (B.6) is nonnegative and has a singularity at

$$
u=\eta^{2}=\frac{r}{\pi^{2}}\left[\log \left(s \varphi\left(N_{0}\right)\right)\right]^{2}
$$

in the case that $\eta>0$.

The RHS of (B.7) can be rewritten in the form

$$
\begin{aligned}
& \frac{1}{2} G_{\eta}(0)+\sum_{\ell=0}^{N_{0}-2}\left[\frac{1}{2} G_{\eta}(\ell)+\frac{1}{2} G_{\eta}(\ell+1)-\int_{\ell}^{\ell+1} G_{\eta}(u) \mathrm{d} u\right] \\
& +\frac{1}{2} G_{\eta}\left(N_{0}-1\right)+\left[G_{\eta}\left(N_{0}-1 / 2\right)-\int_{N_{0}-1}^{N_{0}} G_{\eta}(u) \mathrm{d} u\right] .
\end{aligned}
$$

The first and third terms in (B.9) may be ignored because they are nonnegative. For the second and fourth terms in (B.9), we divide the arguments for their estimates into the following three steps.

Step 1: Estimate of some terms in the sum of the second term in B.9 by the convexity of $G_{\eta}$. Each term in the sum is the error of the trapezoidal approximation of the integral of $G_{\eta}$. The error on the interval $[\ell, \ell+1]$ is 
nonnegative if $G_{\eta}$ is convex on the interval. Therefore, we bound the error from below by zero on the interval where $G_{\eta}$ is convex.

Step 2: Estimate of the other errors in the second term on the intervals in which the singularity $u=\eta^{2}$ is not contained.

Step 3: Estimate of the error in the second term on the interval in which the singularity $u=\eta^{2}$ is contained if it exists and estimate of the fourth term in (B.9). Note that this step is necessary only if $\eta>0$. Therefore, we assume that $\eta>0$ in this step. Then, the singularity is contained in $\left(N_{0}-1, N_{0}\right)$ or $\left(0, N_{0}-1\right)$. In the former case, we have only to estimate the fourth term in this step because all the errors in the second term are already estimated in the previous step.

B.1.1. Step 1. We show a sufficient condition for the convexity of $G_{\eta}$.

Lemma B.4. If $u>r / \pi^{2}$ and $u \neq \eta^{2}$, then the function $G_{\eta}(u)$ given by (B.6) is convex.

Proof. Since

$$
G_{\eta}^{\prime}(u)=-\frac{c_{r} u^{-1 / 2}}{\sinh \left[2 c_{r}(\sqrt{u}-\eta)\right]}
$$

we have

$$
G_{\eta}^{\prime \prime}(u)=\frac{c_{r}}{2 u^{3 / 2}} \frac{\cosh \left[2 c_{r}(\sqrt{u}-\eta)\right]}{\sinh ^{2}\left[2 c_{r}(\sqrt{u}-\eta)\right]}\left(\tanh \left[2 c_{r}(\sqrt{u}-\eta)\right]+2 c_{r} u^{1 / 2}\right) .
$$

If $u>r / \pi^{2}$ and $u \neq \eta^{2}$, then $2 c_{r} u^{1 / 2}>1$ and $G_{\eta}^{\prime \prime}(u)>0$.

We define indexes $\ell_{\mathrm{c}}$ and $\ell_{\mathrm{s}}$ by

$$
\ell_{\mathrm{c}}:=\left\lceil r / \pi^{2}\right\rceil \quad \text { and } \quad \ell_{\mathrm{s}}:=\left\lfloor\eta^{2}\right\rfloor,
$$

respectively. By Lemma B.4 we have

$$
\sum_{\substack{\ell_{c} \leq \ell \leq N_{0}-2, \ell \neq \ell_{\mathrm{s}}}}\left[\frac{1}{2} G_{\eta}(\ell)+\frac{1}{2} G_{\eta}(\ell+1)-\int_{\ell}^{\ell+1} G_{\eta}(u) \mathrm{d} u\right] \geq 0,
$$

which is the desired inequality in Step 1.

B.1.2. Step 2. We start with the estimate

$$
\sum_{\substack{0 \leq \ell \leq \ell_{\mathrm{c}}, \ell \neq \ell_{\mathrm{s}}}}\left[\frac{1}{2} G_{\eta}(\ell)+\frac{1}{2} G_{\eta}(\ell+1)-\int_{\ell}^{\ell+1} G_{\eta}(u) \mathrm{d} u\right] \geq \sum_{\substack{0 \leq \ell \leq \ell_{\mathrm{c}}, \ell \neq \ell_{\mathrm{s}}}} \int_{\ell}^{\ell+1}-G_{\eta}(u) \mathrm{d} u .
$$

By the variable transformation $v=\sqrt{u}$, for $\ell$ with $0 \leq \ell \leq \ell_{\mathrm{c}}$, we have

$$
\begin{aligned}
\int_{\ell}^{\ell+1}-G_{\eta}(u) \mathrm{d} u & =2 \int_{\sqrt{\ell}}^{\sqrt{\ell+1}} \log \left|\tanh \left[c_{r}(v-\eta)\right]\right| v \mathrm{~d} v \\
& \geq 2 \sqrt{\ell_{\mathrm{c}}+1} \int_{\sqrt{\ell}}^{\sqrt{\ell+1}} \log \left|\tanh \left[c_{r}(v-\eta)\right]\right| \mathrm{d} v .
\end{aligned}
$$


Then, we have

$$
\begin{aligned}
& \sum_{\substack{0 \leq \ell \leq \ell_{\mathrm{c}} \\
\ell \neq \ell_{\mathrm{s}}}} \int_{\ell}^{\ell+1}-G_{\eta}(u) \mathrm{d} u \\
\geq & 2 \sqrt{\ell_{\mathrm{c}}+1} \int_{\left[0, \sqrt{\ell_{\mathrm{c}}+1}\right] \backslash\left[\sqrt{\ell_{\mathrm{s}}}, \sqrt{\ell_{\mathrm{s}}+1}\right]} \log \left|\tanh \left[c_{r}(v-\eta)\right]\right| \mathrm{d} v \\
\geq & 2 \sqrt{\ell_{\mathrm{c}}+1} \int_{-\infty}^{\infty} \log \left|\tanh \left[c_{r}(v-\eta)\right]\right| \mathrm{d} v \\
= & 2 \sqrt{\ell_{\mathrm{c}}+1}\left(-\frac{\pi^{2}}{4 c_{r}}\right)=-\pi \sqrt{r} \sqrt{\left[\frac{r}{\pi^{2}}\right]+1} .
\end{aligned}
$$

Inequalities (B.13) and (B.14) give the desired estimate in Step 2.

B.1.3. Step 3. We need to consider the following three cases: (i) $\eta^{2} \in\left(N_{0}-1, N_{0}\right)$, (ii) $\eta^{2} \in\left(N_{0}-2, N_{0}-1\right)$, and (iii) $\eta^{2} \in\left(0, N_{0}-2\right)$. In Case (i), we have $\ell_{\mathrm{s}}=N_{0}-1$ and have only to estimate the fourth term in (B.9):

$$
G_{\eta}\left(N_{0}-1 / 2\right)-\int_{N_{0}-1}^{N_{0}} G_{\eta}(u) \mathrm{d} u
$$

In Cases (ii) and (iii), we have $0 \leq \ell_{\mathrm{s}} \leq N_{0}-2$ and need to estimate the value in (B.15) and the error

$$
\frac{1}{2} G_{\eta}\left(\ell_{\mathrm{s}}\right)+\frac{1}{2} G_{\eta}\left(\ell_{\mathrm{s}}+1\right)-\int_{\ell_{\mathrm{s}}}^{\ell_{\mathrm{s}}+1} G_{\eta}(u) \mathrm{d} u .
$$

For these estimates, we need the following two lemmas.

Lemma B.5. Let $m$ be an integer satisfying $m>1$ and $m-1<\eta^{2}<m+1$, and let $u$ be a real number satisfying $m \leq u \leq m+1$. Then, we have

$$
G_{\eta}(u) \geq \frac{1}{2} \log (m-1)-\log c_{r} .
$$

Proof. Noting that $|\tanh x| \leq|x|$ for any $x \in \mathbf{R}$, we have

$$
\begin{aligned}
G_{\eta}(u) & =-\log \left|\tanh \left[c_{r}(\sqrt{u}-\eta)\right]\right| \geq-\log \left|c_{r}(\sqrt{u}-\eta)\right|=-\log \left|\frac{c_{r}\left(u-\eta^{2}\right)}{\sqrt{u}+\eta}\right| \\
& =\log |\sqrt{u}+\eta|-\log \left|c_{r}\left(u-\eta^{2}\right)\right| \geq \log |\sqrt{m}+\sqrt{m-1}|-\log \left(2 c_{r}\right) \\
& \geq \frac{1}{2} \log (m-1)-\log c_{r} .
\end{aligned}
$$

Lemma B.6. Let $m$ be a nonnegative integer satisfying $m-1<\eta^{2}<m+1$, and let $t_{r}=\tanh \left(2 c_{r}\right) / 2$. Then, we have

$$
-\int_{m}^{m+1} G_{\eta}(u) \mathrm{d} u \geq-\frac{1}{2} \log (m+1)+\log \frac{t_{r}}{4}-\frac{3}{2} .
$$

Proof. Because $\left|\tanh \left(c_{r} x\right)\right| \geq\left|\left(\tanh \left(2 c_{r}\right) / 2\right) x\right|$ for any $x \in \mathbf{R}$ with $|x| \leq 2$, by letting $t_{r}=\tanh \left(2 c_{r}\right) / 2$, we have

$$
-\int_{m}^{m+1} G_{\eta}(u) \mathrm{d} u=\int_{m}^{m+1} \log \left|\tanh \left[c_{r}(\sqrt{u}-\eta)\right]\right| \mathrm{d} u
$$




$$
\geq \int_{m}^{m+1} \log \left|t_{r}(\sqrt{u}-\eta)\right| \mathrm{d} u \geq \int_{\sqrt{m}}^{\sqrt{m+1}} 2 \log |v-\eta| v \mathrm{~d} v+\log t_{r} .
$$

For the estimate of the RHS in (B.17), we use the following indefinite integral:

$$
\int 2 \log |v-\eta| v \mathrm{~d} v=\left(v^{2}-\eta^{2}\right) \log |v-\eta|-\frac{1}{2} v^{2}-\eta v+c^{\prime},
$$

where $c^{\prime}$ is a constant independent of $v$. In the following, we consider the following two cases: (a) $m-1<\eta^{2}<m$ and (b) $m<\eta^{2}<m+1$. Note that Case (a) is void if $m=0$.

$\underline{\text { Case (a) }}$

We have

$$
\begin{aligned}
& \int_{\sqrt{m}}^{\sqrt{m+1}} 2 \log |v-\eta| v \mathrm{~d} v \\
& =\left(m+1-\eta^{2}\right) \log (\sqrt{m+1}-\eta)-\frac{1}{2}(m+1)-\eta \sqrt{m+1} \\
& \quad-\left[\left(m-\eta^{2}\right) \log (\sqrt{m}-\eta)-\frac{1}{2} m-\eta \sqrt{m}\right] \\
& =\log (\sqrt{m+1}-\eta)+\left(m-\eta^{2}\right) \log \frac{\sqrt{m+1}-\eta}{\sqrt{m}-\eta}-\frac{1}{2}-\frac{\eta}{\sqrt{m+1}+\sqrt{m}} \\
& \geq \log (\sqrt{m+1}-\eta)-\frac{1}{2}-\frac{\eta}{\sqrt{m+1}+\sqrt{m}} \\
& \geq \log (\sqrt{m+1}-\eta)-1 .
\end{aligned}
$$

Furthermore, the term $\log (\sqrt{m+1}-\eta)$ is bounded from below as follows:

$$
\begin{aligned}
& \log (\sqrt{m+1}-\eta) \geq \log (\sqrt{m+1}-\sqrt{m}) \\
& =\log \frac{1}{\sqrt{m+1}+\sqrt{m}} \geq-\frac{1}{2} \log (m+1)+\log \frac{1}{2} .
\end{aligned}
$$

From these and (B.17), we have

$$
-\int_{m}^{m+1} G_{\eta}(u) \mathrm{d} u \geq-\frac{1}{2} \log (m+1)+\log \frac{t_{r}}{2}-1 .
$$

\section{Case (b)}

We have

$$
\begin{aligned}
& \int_{\sqrt{m}}^{\sqrt{m+1}} 2 \log |v-\eta| v \mathrm{~d} v=\left(\int_{\sqrt{m}}^{\eta}+\int_{\eta}^{\sqrt{m+1}}\right) 2 \log |v-\eta| v \mathrm{~d} v \\
& =-\left[\left(m-\eta^{2}\right) \log (\eta-\sqrt{m})-\frac{1}{2} m-\eta \sqrt{m}\right] \\
& \quad+\left(m+1-\eta^{2}\right) \log (\sqrt{m+1}-\eta)-\frac{1}{2}(m+1)-\eta \sqrt{m+1} \\
& =\left(m+1-\eta^{2}\right) \log (\sqrt{m+1}-\eta)+\left(\eta^{2}-m\right) \log (\eta-\sqrt{m})-\frac{1}{2}-\frac{\eta}{\sqrt{m+1}+\sqrt{m}}
\end{aligned}
$$




$$
\geq\left(m+1-\eta^{2}\right) \log (\sqrt{m+1}-\eta)+\left(\eta^{2}-m\right) \log (\eta-\sqrt{m})-\frac{3}{2} .
$$

For the estimate of the first and second terms of the last line above, we employ Jensen's inequality

$$
\lambda f\left(x_{1}\right)+(1-\lambda) f\left(x_{2}\right) \geq f\left(\lambda x_{1}+(1-\lambda) x_{2}\right) \quad\left(0 \leq \lambda \leq 1, x_{1}, x_{2}>0\right)
$$

for the function $f(x)=x \log x$ by letting

$$
\lambda=\frac{\sqrt{m+1}+\eta}{\sqrt{m+1}+\sqrt{m}+2 \eta}, \quad x_{1}=\sqrt{m+1}-\eta, \quad x_{2}=\eta-\sqrt{m} .
$$

Then, we have

$$
\begin{aligned}
& \left(m+1-\eta^{2}\right) \log (\sqrt{m+1}-\eta)+\left(\eta^{2}-m\right) \log (\eta-\sqrt{m}) \\
& \geq \log \frac{1}{\sqrt{m+1}+\sqrt{m}+2 \eta} \geq \log \frac{1}{4 \sqrt{m+1}}=-\frac{1}{2} \log (m+1)+\log \frac{1}{4} .
\end{aligned}
$$

From these and (B.17), we have

$$
-\int_{m}^{m+1} G_{\eta}(u) \mathrm{d} u \geq-\frac{1}{2} \log (m+1)+\log \frac{t_{r}}{4}-\frac{3}{2} .
$$

Combining (B.18) in Case (a) and (B.19) in Case (b), we obtain the conclusion of Lemma B.6.

By using Lemmas B.5 and B.6. we finish Step 3.

$\underline{\text { Case (i) }}$

In this case, $\ell_{\mathrm{s}}=N_{0}-1$ holds. By letting $m=N_{0}-1$ in Lemmas B.5 and B.6. and letting $u=N_{0}-1 / 2$ in Lemma B.5, we have

$$
G_{\eta}\left(N_{0}-1 / 2\right)-\int_{N_{0}-1}^{N_{0}} G_{\eta}(u) \mathrm{d} u \geq \frac{1}{2} \log \frac{N_{0}-2}{N_{0}}+\log \frac{t_{r}}{4 c_{r}}-\frac{3}{2} .
$$

Thus, the value in (B.15) is bounded from below by a constant independent of $N_{0}$ and $\eta$.

Case (ii)

In this case, $\ell_{\mathrm{s}}=N_{0}-2$ holds. First, we can estimate the value in (B.15) in the same manner as (B.20) in Case (i). Next, we estimate the value in (B.16). By letting $m=\ell_{\mathrm{s}}$ in Lemmas B.5 and B.6, and letting $u=\ell_{\mathrm{s}}$ and $u=\ell_{\mathrm{s}}+1$ in Lemma B.5, we have

$$
\frac{1}{2} G_{\eta}\left(\ell_{\mathrm{s}}\right)+\frac{1}{2} G_{\eta}\left(\ell_{\mathrm{s}}+1\right)-\int_{\ell_{\mathrm{s}}}^{\ell_{\mathrm{s}}+1} G_{\eta}(u) \mathrm{d} u \geq \frac{1}{2} \log \frac{\ell_{\mathrm{s}}-1}{\ell_{\mathrm{s}}+1}+\log \frac{t_{r}}{4 c_{r}}-\frac{3}{2} .
$$

Thus, the value in (B.16) is bounded from below by a constant independent of $N_{0}$ and $\eta$.

Case (iii) 
In this case, $0 \leq \ell_{\mathrm{s}} \leq N_{0}-3$ holds. First, we estimate the value in (B.16). In the case that $\ell_{\mathrm{s}}>1$, we can estimate it in the same manner as (B.21) in Case (ii). In the case that $0 \leq \ell_{\mathrm{s}} \leq 1$, by Lemma B.6 with $m=\ell_{\mathrm{s}}$, we have

$$
\begin{aligned}
& \frac{1}{2} G_{\eta}\left(\ell_{\mathrm{s}}\right)+\frac{1}{2} G_{\eta}\left(\ell_{\mathrm{s}}+1\right)-\int_{\ell_{\mathrm{s}}}^{\ell_{\mathrm{s}}+1} G_{\eta}(u) \mathrm{d} u \\
& \geq-\int_{\ell_{\mathrm{s}}}^{\ell_{\mathrm{s}}+1} G_{\eta}(u) \mathrm{d} u \geq-\frac{1}{2} \log 2+\log \frac{t_{r}}{4}-\frac{3}{2} .
\end{aligned}
$$

Thus, the value in (B.16) is bounded from below by a constant independent of $N_{0}$ and $\eta$. Next, we estimate the value in B.15). Because $G_{\eta}$ is monotone decreasing on $\left(\eta^{2}, \infty\right)$, we have

$$
\begin{aligned}
& G_{\eta}\left(N_{0}-1 / 2\right)-\int_{N_{0}-1}^{N_{0}} G_{\eta}(u) \mathrm{d} u \geq G_{\eta}\left(N_{0}-1 / 2\right)-G_{\eta}\left(N_{0}-1\right) \\
& =-\log \left|\tanh \left[c_{r}\left(\sqrt{N_{0}-1 / 2}-\eta\right)\right]\right|+\log \left|\tanh \left[c_{r}\left(\sqrt{N_{0}-1}-\eta\right)\right]\right| .
\end{aligned}
$$

If we regard the function given by the RHS in (B.22) as a function of $\eta$, it is monotone decreasing because its derivative with respect to $\eta$ satisfies

$$
\frac{c_{r}}{\sinh \left[2 c_{r}\left(\sqrt{N_{0}-1 / 2}-\eta\right)\right]}-\frac{c_{r}}{\sinh \left[2 c_{r}\left(\sqrt{N_{0}-1}-\eta\right)\right]}<0 .
$$

Therefore, we have

$$
\begin{aligned}
& -\log \left|\tanh \left[c_{r}\left(\sqrt{N_{0}-1 / 2}-\eta\right)\right]\right|+\log \left|\tanh \left[c_{r}\left(\sqrt{N_{0}-1}-\eta\right)\right]\right| \\
& \geq-\log \left|\tanh \left[c_{r}\left(\sqrt{N_{0}-1 / 2}-\sqrt{N_{0}-2}\right)\right]\right|+\log \left|\tanh \left[c_{r}\left(\sqrt{N_{0}-1}-\sqrt{N_{0}-2}\right)\right]\right| \\
& \geq-\log \left|c_{r}\left(\sqrt{N_{0}-1 / 2}-\sqrt{N_{0}-2}\right)\right|+\log \left|\left(\tanh c_{r}\right)\left(\sqrt{N_{0}-1}-\sqrt{N_{0}-2}\right)\right| \\
& =\log \left|\frac{\sqrt{N_{0}-1}-\sqrt{N_{0}-2}}{\sqrt{N_{0}-1 / 2}-\sqrt{N_{0}-2}}\right|+\log \frac{\tanh c_{r}}{c_{r}} \\
& =\log \left|\frac{\sqrt{N_{0}-1 / 2}+\sqrt{N_{0}-2}}{\sqrt{N_{0}-1}+\sqrt{N_{0}-2}}\right|+\log \frac{2 \tanh c_{r}}{3 c_{r}} .
\end{aligned}
$$

Thus, the value in (B.15) is bounded from below by a constant independent of $N_{0}$ and $\eta$.

From the estimates in the three cases above, Step 3 is completed. Thus, LemmaB.3 is proven.

\section{ACKNOWLEDGMENT}

T. Okayama and M. Sugihara are supported by the grant-in-aid of Japan Society for the Promotion of Science (KAKENHI Grant Numbers: JP24760060 (Okayama), JP25390146 (Sugihara)). 


\section{REFERENCES}

[1] H. G. Burchard and K. Hollig, n-width and entropy of $H^{p}$-classes in $L_{q}(-1,1)$, SIAM J. Math. Anal. 16 (1985), 405-421.

[2] T. H. Ganelius, Rational approximation in the complex plane and on the line, Annales Academiæ Scientiarum Fennicæ Series A. I. Mathematica 2 (1976), 129-145.

[3] A. P. Jang and S. Haber, Numerical indefinite integration of functions with singularities, Math. Comp. 70 (2001), 205-221.

[4] J. Lund and K. L. Bowers, Sinc methods for quadrature and differential equations, SIAM, Philadelphia, 1992.

[5] F. Stenger, Optimal convergence of minimum norm approximations in $H_{p}^{*}$, Numer. Math. 29 (1978), 345-362.

[6] F. Stenger, Numerical methods based on sinc and analytic functions, Springer-Verlag, New York, 1993.

[7] F. Stenger, Summary of sinc numerical methods, J. Comput. Appl. Math. 121 (2000), 379420.

[8] F. Stenger, Handbook of sinc numerical methods, CRC Press, Boca Raton, 2011.

[9] M. Sugihara, Near optimality of the sinc approximation, Math. Comp. 72 (2003), 767-786.

[10] K. Tanaka, M. Sugihara and K. Murota, Function classes for successful DE-Sinc approximations, Math. Comp. 78 (2009), 1553-1571.

[11] T. Ushima, K. Tanaka, T. Okayama and M. Sugihara, A function approximation formula using the Ganelius sampling points, submitted (in Japanese).

[12] K. Wilderotter, $n$-widths of $H^{p}$-spaces in $L_{q}(-1,1)$, J. Complexity 8 (1992), 324-335.

Department of Mathematical Engineering, Faculty of Engineering, Musashino UniVERsity, 3-3-3, ARIAKe, Koto-KU, TOKYo 135-8181, JAPAN

E-mail address: ketanaka@musashino-u.ac.jp

Department of Systems Engineering, Graduate School of Information Sciences, Hiroshima City University, 3-4-1, Ozuka-higashi, Asaminami-Ku, Hiroshima 731-3194, Japan

E-mail address: okayama@hiroshima-cu.ac.jp

Department of Physics and Mathematics, College of Science and Engineering, Aoyama Gakuin University 5-10-1, Fuchinobe, Chuo-ku, Sagaminara-Shi, Kanagawa 252-5258, Japan

E-mail address: sugihara@gem.aoyama.ac.jp 